\title{
ASYMPTOTISCHE ENTWICKLUNGEN, TEICHMÜLLER-WITTICHSCHER VERZERRUNGSSATZ UND KOMPAKTHEIT IN KLASSEN QUASIKONFORMER ABBILDUNGEN
}

\author{
HEINRICH RENELT
}

Analog zu den konformen Abbildungen (z. B. der Klassen $S$ und $\Sigma$ ) können auch bei quasikonformen Abbildungen bzw. schlichten Lösungen gleichmäßig elliptischer Differentialgleichungssysteme asymptotische Entwicklungen der Form

$$
\text { (0.1) } f(z)=f\left(z_{0}\right)+a \cdot\left(z-z_{0}\right)+b\left(\overline{z-z_{0}}\right)+o\left(\left|z-z_{0}\right|\right) \text { mit } \lim _{z \rightarrow z_{0}} \frac{o\left(\left|z-z_{0}\right|\right)}{z-z_{0}}=0
$$

in einem bestimmten Regularitätspunkt $z_{0}$ von $f(z)$ (bei entsprechenden Modifizierungen von (0.1) im Falle $z_{0}=\infty$ und/oder $f\left(z_{0}\right)=\infty$ ) als Normierungen verwendet werden. Hierzu wird der lineare Bestandteil in (0.1) vorgeschrieben, was natürlich wie auch bei konformen Abbildungen nicht ganz beliebig geschehen kann. Solche Normierungen treten in vielen physikalischen Problemen auf (siehe z. B. [9] und dort genannte Literatur).

Von speziellen Fällen abgesehen ist nichtsdestoweniger bisher die Frage unbehandelt geblieben, wann so normierte quasikonforme Abbildungen eine (nichtleere) kompakte Menge bilden. Dies ist (nach einigen Vorbereitungen im 1. Abschnitt) Gegenstand des 2. Abschnitts dieser Mitteilung (Sätze 1 und 2). Dabei spielt der Teichmüller-Wittichsche Verzerrungssatz (siehe [11], [13]) in seiner erweiterten Form (siehe [3], [7]) eine wichtige Rolle. In den folgenden beiden Abschnitten werden unter (gegenüber der Voraussetzung des Teichmüller-Wittichschen Verzerrungssatzes, siehe (1.6) unten) verschärften Bedingungen (siehe (1.7), (1.8) unten) verschärfte Formen des erweiterten Teichmüller-Wittichschen Verzerrungssatzes bewiesen (Sätze 4, 4' und 6), mit deren Hilfe die zugehörigen asymptotischen Entwicklungen genauer beschrieben werden können (Sätze 3 und 5). Unter der im Satz 5 zu Grunde gelegten Bedingung ergeben sich dabei sehr ähnliche Verhältnisse wie in der Klasse $\Sigma(G)$ der konformen hydrodynamisch normierten Abbildungen eines Gebietes $G$ mit $\infty \in G$.

Aus beweistechnischen Gründen wird hier (außer bei Satz 4') der Fall $z_{0}=$ $f\left(z_{0}\right)=\infty$ behandelt. Mittels Möbiustransformationen und affiner Transformationen kann man den Fall beliebiger $z_{0}$ und $f\left(z_{0}\right)$ hierauf zurückführen. 


\section{Vorbereitende Betrachtungen}

$G$ sei ein Gebiet der vollen $z$-Ebene $E=E_{z}, z=x+i y$, mit $\left\{|z| \geqq R_{0}\right\} \subset G, R_{0}$ eine feste positive endliche Zahl. Die Funktion $\mu(z)$ sei eine für alle $z \in E_{z}$ definierte meßbare Funktion mit

$$
\|\mu\|_{\infty}=\underset{|z|<\infty}{\operatorname{ess} \sup }|\mu(z)|=q_{0}<1 \quad \text { und } \quad|\mu(\infty)|<1 .
$$

$D$ sei eine (nicht notwendig echte) Teilmenge von $G$, die $\left\{|z| \geqq R_{0}\right\}$ im Innern enthält. $\mathscr{M}(\mu)$ sei die Klasse aller (schlichten) quasikonformen Abbildungen $f$ von $G$ mit

$$
\begin{gathered}
\left|f_{\bar{z}}\right| \leqq|\mu(z)| \cdot\left|f_{z}\right| \quad \text { für f. a. (= fast alle) } \quad z \in G, \\
\qquad f_{\bar{z}}=\mu(z) \cdot \overline{f_{z}} \text { für f. a. } \quad z \in D .
\end{gathered}
$$

$\mathscr{M}(\mu)$ ist natürlich nicht leer (siehe z. B. [6] und dort genannte Literatur). Nach bekannten Konvergenzsätzen für quasikonforme Abbildungen (siehe [8], S. 74; [10]) sowie auf Grund der schwachen Kompaktheit beschränkter Mengen in $L_{2}$ (im Hinblick auf die Gültigkeit von (1.3) für die Grenzfunktionen) ist $\mathscr{M}(\mu)$ abgeschlossen in der Menge aller $Q$-quasikonformen Abbildungen von $G$ mit $Q=\left(1+q_{0}\right) /\left(1-q_{0}\right)$ bezüglich punktweiser Konvergenz unter Beachtung der Tatsache, daß punktweise Konvergenz einer Folge quasikonformer Abbildungen von $G$ gegen eine quasikonforme Abbildung von $G$ notwendigerweise gleichmäßig in jedem kompakten Teilgebiet von $G$ sein muß (siehe [8], S. 74). $\mathscr{M}(\mu)$ ist also (erst recht) auch abgeschlossen bezüglich gleichmäßiger Konvergenz in kompakten Teilgebieten von $G$ (=lokal gleichmäßige Konvergenz in $G$ ). Unter Konvergenz in $\mathscr{M}(\mu)$ oder in irgendeiner der unten genannten Klassen quasikonformer $\mathrm{Ab}$ bildungen soll nun stets die lokal gleichmäßige Konvergenz verstanden werden.

Die Affinität

$$
F=\frac{1}{1-|\mu(\infty)|^{2}} \cdot f-\frac{\mu(\infty)}{1-|\mu(\infty)|^{2}} \cdot \bar{f}
$$

führt jedes $f \in \mathscr{M}(\mu)$ über in ein $F \in \mathscr{M}\left(\mu_{0}\right)$ mit

$$
\mu_{0}(z)= \begin{cases}(\mu(z)-\mu(\infty)) /(1-\overline{\mu(\infty)} \mu(z)) & \text { für } \quad z \in D \\ (|\mu(z)|+|\mu(\infty)|) /(1+|\mu(\infty) \mu(z)|) & \text { sonst. }\end{cases}
$$

Für das Regularitätsverhalten der $f \in \mathscr{M}\left(\mu_{0}\right)$ in $\infty$ ist natürlich nicht $\mu_{0}(\infty)=0$, sondern allein das Verhalten von $\mu_{0}$ in einer Umgebung von $\infty$ entscheidend. Eine in gewissem Sinne (siehe [11]; [8], S. 220) nicht weiter abschwächbare Voraussetzung für Regularität ist die Voraussetzung des Teichmüller-Wittichschen Verzerrungssatzes (Voraussetzung TW):

(1.6) $\int_{\left\{|z|>R_{0}\right\}} \frac{|\mu(z)-\mu(\infty)|}{|z|^{2}} d \sigma_{z}<\infty \quad\left(d \sigma_{z}\right.$ das ,Flächenelement” in der $z$-Ebene $)$. 
Voraussetzung TW ist offenbar genau dann erfüllt, wenn für das zugehörige $\mu_{0}$ gilt

$$
\int_{\left\{|z|>R_{0}\right\}} \frac{\left|\mu_{0}(z)\right|}{|z|^{2}} d \sigma_{z}<\infty .
$$

Weiterhin sollen hier noch solche $\mu(z)$ betrachtet werden, die die folgenden stärkeren Bedingungen

$$
\mu(z)-\mu(\infty) \in L_{p_{0}}, p_{0} \geqq 2 \text { und } \lim _{z \rightarrow \infty} \mu(z)=\mu(\infty)
$$

(Voraussetzung $\left.\left(L_{p}\right)\right)$ und

$$
\mu(z)-\mu(\infty) \in L_{q} \quad \text { mit } \quad 1 \leqq q<2
$$

(Voraussetzung $\left(L^{q}\right)$ ) erfüllen (siehe auch Abschnitt 3, Bemerkung 3 unten). Wenn z. B. $\mu(z)$ in $\infty$ hölderstetig ist, d. h. wenn gilt

$$
|\mu(z)-\mu(\infty)| \leqq \frac{c}{|z|^{\alpha}} \text { für alle } z \text { mit positiven Konstanten } c, \alpha,
$$

so ist $\alpha>0$ hinreichend für (1.7) und $\alpha>1$ hinreichend für (1.8), und zwar gilt dann (1.7), (1.8) für jedes $p_{0}$ bzw. $q$, das größer als $2 / \alpha$ ist. Wegen (1.1), das im folgenden bei TW, $\left(L_{p}\right)$ und $\left(L^{q}\right)$ stets gleichzeitig mit vorausgesetzt wird, gilt (man beachte, daß $(\mu-\mu(\infty)) /(1-\overline{\mu(\infty)} \cdot \mu)$ eine lineare Transformation des $\mu$ Einheitskreises in sich darstellt):

(1.10) Wenn $\mu(z)-\mu(\infty) \in L_{r}$ ist mit $r \geqq 1$, so ist auch $\mu(z)-\mu(\infty) \in L_{s}$ für jedes $s \geqq r$, und außerdem ist dann $|\mu(\infty)| \leqq q_{0}$.

Sei $\mathscr{Q}(\mu, b)$ mit $b \in G, b \neq \infty$, die Teilmenge der $f \in \mathscr{M}(\mu)$, für die $f(b)=b$ gilt und die eine Entwicklung

$$
f(z)=z+\mu(\infty) \bar{z}+\varepsilon_{f}(z) \text { mit } \lim _{z \rightarrow \infty} \frac{\varepsilon_{f}(z)}{z}=0
$$

besitzen (insbesondere ist dann $f(\infty)=\infty$ ).

\section{Der Fall TW}

Es gilt der folgende

Satz 1. Wenn $\mu$ die Voraussetzung $T W$ erfüllt, so ist $\mathscr{2}(\mu, b)$ nicht leer und kompakt in sich.

Beweis. Es genügt natürlich, Satz 1 im Falle $\mu(\infty)=0$ zu beweisen. Zum Nachweis von $\mathscr{Q}(\mu, b) \neq \emptyset$ betrachte man eine Folge

$$
\mu_{n}(z)=\mu(z) \text { für }|z| \leqq R_{n}, \quad \mu_{n}(z)=0 \quad \text { sonst }
$$


mit $R_{n} \rightarrow \infty$. Sei $H_{n}(z)$ die schlichte Abbildung der vollen $z$-Ebene mit $H_{n \bar{z}}=$ $\mu_{n} \overline{H_{n z}}, H_{n}(z)=z+a_{0 n}+a_{1 n} / z+\ldots$ für $|z|>R_{n}, H_{n}(0)=0$ (siehe z. B. [6]). Nach [8], S. 232 (Satz 6.1 und die anschließenden Bemerkungen), angewendet auf $g_{n}(z)=$ $1 / H_{n}(1 / z)$, folgt die Existenz eines $R^{*}<\infty$ z. B. mit $(1 / 2) R^{*} \leqq\left|H_{n}(z)\right| \leqq 2 R^{*}$ für alle $n$ und alle $z$ mit $|z|=R^{*}$. Folglich bilden die $H_{n}$ eine kompakte Menge, und eine Teilfolge der $H_{n}$ konvergiert gegen eine schlichte quasikonforme $\mathrm{Ab}$ bildung $H(z)$ der vollen $z$-Ebene, die auf Grund der obigen Bemerkungen zur Abgeschlossenheit von $\mathscr{M}(\mu)$ Lösung von $H_{\bar{z}}=\mu \bar{H}_{z}$ ist. Wieder nach [8], S. 232 besitzt $H$ jedenfalls eine Entwicklung $H(z)=c z+\varepsilon(z)$ mit $\varepsilon(z) / z \rightarrow 0$ für $z \rightarrow \infty$, $c \neq 0$. Der Fall $c=1$ ist dann eine Konsequenz aus der Gleichmäßigkeitsaussage in [8], S. 232, 1. Bemerkung, und der lokal gleichmäßigen Konvergenz der $H_{n}$. Also gehört $H(z)-(H(b)-b)$ zu $2(\mu, b)$. Nach demselben Muster ergibt sich auch die Abgeschlossenheit von $2(\mu, b)$ in $\mathscr{M}(\mu)$.

Sei nun $\Sigma(\mu, b)$ die Menge aller quasikonformen Abbildungen $f$ von $G$ mit $f(b)=b$, die (1.2) erfüllen und eine Entwicklung (1.11) besitzen. Wegen $\Sigma(\mu, b) \supset \mathscr{Q}(\mu, b)$ ist $\Sigma(\mu, b)$ nicht leer. Satz 1 ist offenbar bewiesen, wenn gezeigt ist der

Satz 2. Wenn $\mu$ die Voraussetzung $T W$ und $\mu(\infty)=0$ erfüllt, so ist $\Sigma(\mu, b)$ kompakt in sich.

Beweis von Satz 2. Zunächst ist aus den oben genannten Gründen natürlich $\Sigma(\mu, b)$ abgeschlossen in der Menge der $Q$-quasikonformen Abbildungen von G. Gegeben sei nun eine Folge $f_{n} \in \Sigma(\mu, b)$, und es sei $g_{n}(t)=1 / f_{n}(1 / t)$. Sei $h_{n}$ eine konforme Koebe-normierte Abbildung von $g_{n}\left(\left\{|t|<1 / R_{0}\right\}\right)$ auf einen Kreis $\left\{|w|<r_{n}\right\}$. Durch Spiegelung an den Kreisen $\left\{|t|=1 / R_{0}\right\}$ und $\left\{|w|=r_{n}\right\}$ kann $g_{n}^{*}(t)=h_{n} \circ g_{n}(t)$ in die gesamte $t$-Ebene $Q$-quasikonform fortgesetzt werden mit $g_{n}^{*}(0)=0, g_{n}^{*}(\infty)=\infty$ für alle $n$. Nach [8], S. 232 folgt dann wieder die Existenz eines $s_{0}>0 \quad$ z. B. mit $(1 / 2) s \leqq\left|g_{n}^{*}(t)\right| \leqq 2 s$ für $|t|=s \leqq s_{0}$ und alle $n$, was zusammen mit $g_{n}^{*}(0)=0, g_{n}^{*}(\infty)=\infty$ die Kompaktheit der $g_{n}^{*}$ und damit auch $r_{n} \geqq r>0$ mit einem festen $r$ liefert. Aus bekannten Verzerrungssätzen für Koebe-normierte konforme Abbildungen (siehe z. B. [4], S. 102), angewendet auf die Umkehrabbildungen der $h_{n}$, ergibt sich dann auch die Existenz eines $R>R_{0}$ und zweier positiver Konstanten $c_{1}, c_{2}$ mit $c_{1} R \leqq\left|f_{n}(z)\right| \leqq c_{2} R$ für alle $z$ mit $|z|=R$ und alle $n$, wobei noch ohne Beschränkung der Allgemeinheit $c_{1} R>|b|$ angenommen werden kann. Zusammen mit $f_{n}(b)=b, f_{n}(\infty)=\infty$ liefert dies die lokal gleichmäßige Konvergenz einer Teilfolge der $f_{n}$ in $G$ gegen eine $Q$-quasikonforme Abbildung von $G$, womit Satz 2 und damit auch Satz 1 bewiesen ist.

Bemerkung 1. Wenn in der Definition von $\mathscr{M}(\mu)(1.2),(1.3)$ ersetzt werden durch

$$
\left|f_{\bar{z}}\right| \leqq(|v|+|\mu|) \cdot\left|f_{z}\right| \quad \text { für f. a. } \quad z \in G \quad \text { mit } \quad|\mu|+|v| \leqq q_{0}<1 \quad \text { für alle } z,
$$

$$
f_{\bar{z}}=v f_{z}+\mu \overline{f_{z}} \quad \text { für f. a. } \quad z \in D,
$$


so läßt sich durch Affinitäten in der $f$ - und $z$-Ebene $v(\infty)=\mu(\infty)=0$ erreichen. Für die (bereits transformierten) $v, \mu$ hat man dann als Voraussetzung TW zu fordern

$$
\int_{\left\{|z|>R_{0}\right\}} \frac{|v(z)|+|\mu(z)|}{|z|^{2}} d \sigma_{z}<\infty .
$$

Für die Menge $\mathscr{Q}(v, \mu, b)$ der quasikonformen Abbildungen $f$ von $G$ mit $f(b)=b$, die $\left(1.2^{\prime}\right)$ und $\left(1.3^{\prime}\right)$ erfüllen und eine Entwicklung

$$
\left(1.11^{\prime}\right) f(z)=z+\varepsilon_{f}(z) \text { mit } \quad \varepsilon_{f}(z) / z \rightarrow 0 \text { für } \quad z \rightarrow \infty \quad(v(\infty)=\mu(\infty)=0)
$$

besitzen, und $\Sigma(|v|+|\mu|, b)$ gelten dann wieder die Sätze 1 und 2, wobei man nur im Beweis von Satz $1 H_{n \bar{z}}=\mu_{n} \overline{H_{n z}}, H_{\bar{z}}=\mu \overline{H_{z}}$ durch $H_{n \bar{z}}=v_{n} H_{n z}+\mu_{n} \overline{H_{n z}}, H_{\bar{z}}=$ $v H_{z}+\mu \bar{H}_{z}$ mit analog zu (2.1) definierten $v_{n}, \mu_{n}$ zu ersetzen hat. Einsetzen der oben genannten Affinitäten in $\left(1.11^{\prime}\right)$ liefert dann die Entwicklung

$\left(11.1^{\prime \prime}\right) \quad f(z)=z+[v(\infty)+\mu(\infty)] \bar{z}+\varepsilon_{f}(z)$ mit $\quad \varepsilon_{f}(z) / z \rightarrow 0 \quad$ für $\quad z \rightarrow \infty$

für die $f \in \mathscr{Q}(v, \mu, b)$ und natürlich auch die Gültigkeit von Satz 1, wenn nicht notwendig $v(\infty)=\mu(\infty)=0$ erfüllt ist.

\section{Der Fall $\left(L_{p}\right)$}

Wenn nicht nur TW, sondern sogar $\left(L_{p}\right)$ erfüllt ist, so hat man als Ergänzung zu den Sätzen 1 und 2 den folgenden

Satz 3. Die Funktionen $\mu^{0}(z), \mu(z)$ mögen die Voraussetzung $\left(L_{p}\right)$ erfüllen, und es sei $\mu^{0}(\infty)=0$. Für alle

$$
f(z)=z+\mu(\infty) \bar{z}+\varepsilon_{f}(z) \in \mathscr{Q}(\mu, b) \quad(\mu(\infty) \text { nicht notwendig }=0)
$$

und alle

gilt dann

$$
f(z)=z+\varepsilon_{f}(z) \in \Sigma\left(\mu^{0}, b\right)
$$

$$
\lim _{z \rightarrow \infty} \varepsilon_{f}(z) \cdot|z|^{-\gamma}=0 \quad \text { für jedes feste } \quad \gamma>1-\left(2 / p_{0}\right)
$$

gleichmäßig für alle $f \in \mathscr{Q}(\mu, b)$ bzw. für alle $f \in \Sigma\left(\mu^{0}, b\right)$.

Der Beweis des Satzes 3 beruht auf einer Verschärfung des (erweiterten) Teichmüller-Wittichschen Verzerrungssatzes, nämlich dem

Satz 4. Die Funktion $\mu(z)$ erfülle (1.1), (1.7) und $\mu(\infty)=0$. Eine beschränkte Menge $Z_{1} \subset G$ und ein $p>2$ mit $p \geqq p_{0}$ seien beliebig, aber fest vorgegeben. Dann gibt es zwei feste endliche positive Konstanten $c_{1}, c_{2}$ und zu jeder quasikonformen 
Abbildungen $f(z)$ von $G$ mit $f(\infty)=\infty$, die (1.2) erfüllt, eine endliche Konstante $c_{f} \neq 0$, so daß gilt

$$
\left|\frac{f(z)-f\left(z_{1}\right)}{z}-c_{f}\right| \leqq\left|c_{f}\right|\left[c_{1}|z|^{-2 / p}+c_{2}|z|^{-1}\right]
$$

für alle $z \in G$ und für alle $z_{1} \in Z_{1}$.

Beweis von Satz 4. $C(s)$ sei die Norm der Hilberttransformation

$$
T h(z)=-\frac{1}{\pi} \int \frac{h(\zeta)}{(\zeta-z)^{2}} d \sigma_{\zeta} \quad \text { in } \quad L_{s}=L_{s}(E),
$$

$E$ die (in Zusammenhang mit Integrierbarkeit oder Meßbarkeit stets endliche und sonst volle) $z$-bzw. $\zeta$-Ebene, $s>1$ (Integrale, bei denen kein Integrationsgebiet angegeben ist, sind stets über den gesamten Träger des Integranden zu berechnen). Wegen $C(s) \rightarrow 1$ für $s \rightarrow 2$ (siehe [5], [2]) gibt es ein $s$ mit $s>2$, das im weiteren festgehalten werden soll, mit

$$
C(s) \cdot q_{0}=k_{0}<1 .
$$

Im Falle $p_{0}>2$ in (1.7) sei außerdem $s \leqq p_{0}$ gewählt. Wegen (1.7) und $\mu(\infty)=0$

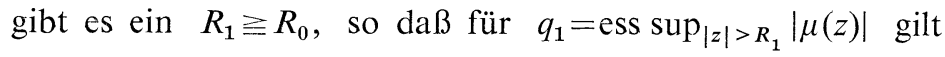

(3.3) $C(p) \cdot q_{1}=k_{1}<1$ für $p=p_{0}$ im Falle $p_{0}>2, \quad p=s$ im Falle $p_{0}=2$.

Für $s$ und $p$ gilt dann $p \geqq s>2$. Sei nun $f$ irgendeine quasikonforme Abbildung von $G$ mit $f(\infty)=\infty$, die (1.2) (mit $\mu(\infty)=0$ ) erfüllt und sei $\mu_{f}(z)=f_{\bar{z}} / f_{z}$ für $z \in G, \mu_{f}(z)=0$ sonst. Sei weiter $\mu_{0}(z)=\mu_{f}(z)$ für $|z| \leqq R_{1}, \mu_{0}(z)=0$ sonst, und sei $\mu_{1}(z)=\mu_{f}(z)$ für $|z| \geqq R_{1}, \mu_{1}(z)=0$ sonst. Wegen (1.7), (3.3) hat

$$
h_{1}=\mu_{1}+\mu_{1} T h_{1}
$$

eine eindeutig bestimmte Lösung $h_{1} \in L_{p}$. Ebenso hat auch wegen (3.2) und $\mu_{0} \equiv 0$ für $|z|>R_{1}$

$$
h_{0}=\mu_{0}\left(1+T h_{1}\right)+\mu_{f} T h_{0}
$$

eine eindeutig bestimmte Lösung $h_{0} \in L_{s}$. Für $h=h_{0}+h_{1}$ gilt dann

$$
h=\mu_{f}+\mu_{f} T h \text {. }
$$

Sei weiter

$$
P_{0} g(z)=-\frac{1}{\pi} \int g(\zeta)\left[\frac{1}{\zeta-z}-\frac{1}{\zeta}\right] d \sigma_{\zeta}, \quad g \in L_{r}, r>2 .
$$

Mit $P_{0} h=P_{0} h_{0}+P_{0} h_{1}$ wird dann

$$
H_{f}(z)=z+P_{0} h(z)
$$

eine in der ganzen Ebene schlichte Lösung des Beltramischen Differentialgleichungssystems

$$
\left(H_{f}\right)_{\bar{z}}=\mu_{f} \cdot\left(H_{f}\right)_{z}
$$


mit $H_{f}(0)=0, H_{f}(\infty)=\infty$. Denn (3.9) folgt sofort aus $\left(P_{0} h\right)_{\bar{z}}=h,\left(P_{0} h\right)_{z}=T h$ und (3.6). Außerdem ist (vgl. [1], Kap. V A)

$$
\left|P_{0} h(z)\right| \leqq\left|P_{0} h_{0}\right|+\left|P_{0} h_{1}\right| \leqq K_{s}\left\|h_{0}\right\|_{L_{s}} \cdot|z|^{1-(2 / s)}+K_{p}\left\|h_{1}\right\|_{L_{p}} \cdot|z|^{1-(2 / p)},
$$

wobei $K_{s}, K_{p}$ Konstanten sind, die nur von $s$ bzw. $p$ abhängen. Die Schlichtheit von $H_{f}$ folgt dann unter Verwendung der Existenz mindestens einer in der ganzen $z$-Ebene schlichten Lösung $w(z)$ von (3.9) mit $w(\infty)=\infty$ (siehe z. B. [5]) aus $H_{f}(z)=\omega \circ w(z)$ mit einer ganzen analytischen Funktion $\omega(w)$, dem Argumentprinzip, (3.8) und (3.10).

Aus (3.10), (3.4), (3.5) erhält man weiter die Abschätzung

$$
\left|P_{0} h(z)\right| \leqq K_{p} K_{0}\left\|\mu_{f}\right\|_{L_{p}} \cdot|z|^{1-(2 / p)}+K_{s} K_{0}\left(\left\|\mu_{0}\right\|_{L_{s}}+\left\|\mu_{0} T h_{1}\right\|_{L_{s}}\right) \cdot|z|^{1-(2 / s)}
$$

mit $K_{0}=1 /\left(1-\max \left(k_{0}, k_{1}\right)\right)$. Unter Beachtung der Hölderschen Ungleichung erhält man dann weiter

$$
\left\|\mu_{0} T h_{1}\right\|_{L_{s}} \leqq q_{0}\left\|T h_{1}\right\|_{\left.L_{s}\left\{|z| \leqq R_{1}\right\}\right)}=k_{0}\left(\pi R_{1}^{2}\right)^{(p-s) / p s} \cdot\left\|h_{1}\right\|_{L_{p}} .
$$

Wegen $\left|\mu_{f}(z)\right| \leqq|\mu(z)|$ für alle $z$ gilt dann

$$
\begin{aligned}
\left|P_{0} h(z)\right| & \leqq K_{p} K_{0}\|\mu\|_{L_{p}} \cdot|z|^{1-(2 / p)}+K_{s} K_{0}\left[\|\mu\|_{L_{s}\left(\left\{|z| \leqq R_{1}\right\}\right)}+\right. \\
& \left.+k_{0} K_{0}\left(\pi R_{1}^{2}\right)^{(p-s) / p s} \cdot\|\mu\|_{L_{p}}\right] \cdot|z|^{1-(2 / s)}= \\
& =d_{0}|z|^{1-(2 / s)}+d_{1}|z|^{1-(2 / p)} \quad \text { für alle } z
\end{aligned}
$$

mit Konstanten $d_{0}, d_{1}$, die nur von $\mu, R_{1}, p$ und $s$ abhängen.

Da $H_{f}(z)$ und $f(z)$ in $G$ Lösung ein und desselben Systems (3.9) sind, gibı es eine schlichte konforme Abbildung $\omega_{f}(\cdot)$ von $H_{f}(G)$ mit $\omega_{f}(\infty)=\infty$ und

$$
f(z)=\omega_{f} \circ H_{f}(z) .
$$

Die Abbildung $\omega_{f}$ besitzt eine Entwicklung

$$
\omega_{f}(H)=c H+a_{0}+a_{1} / H+\ldots
$$

mit natürlich von $f$ abhängenden Koeffizienten $c \neq 0$ und $a_{i}, i=0,1,2, \ldots$ Betrachtet man nun die Gesamtheit dieser $f$ und die Menge der zugehörigen $H_{f}$, so bilden diese $H_{f}$ eine kompakte Menge in der Menge der schlichten $Q$-quasikonformen Abbildungen von $G$ (wegen $H_{f}(0)=0, H_{f}(\infty)=\infty$, (3.9), (3.8) und (3.12)). Folglich gibt es zu jeder festen beschränkten Menge $Z_{1} \subset G$ eine Konstante $M\left(Z_{1}\right)<\infty$ mit $\left|H_{f}(z)\right| \leqq M\left(Z_{1}\right)$ für alle $z \in Z_{1}$ und alle $H_{f}$. Außerdem gilt wegen $(3.12)$

$$
H_{f}\left(\left\{|z|>R_{0}\right\}\right) \supset\left\{|H|>R_{0}^{*}\right\} \quad \text { mit } \quad R_{0}^{*}=R_{0}+d_{0} R_{0}^{1-(2 / s)}+d_{1} R_{0}^{1-(2 / p)} .
$$

Folglich bilden auch die konformen hydrodynamisch normierten Abbildungen

$$
\omega_{f}(H) / c-a_{0} / c=H+\frac{a_{1} / c}{H}+\ldots
$$


eine kompakte Menge schlichter konformer Abbildungen, und somit ist

$$
\left|\left(f\left(z_{1}\right)-a_{0}\right) / c\right|=\left|\left(\omega_{f} \circ H_{f}\left(z_{1}\right)-a_{0}\right) / c\right| \leqq L \text { für alle } z_{1} \in Z_{1} \text { und alle } f
$$

mit einer festen endlichen Konstanten $L=L\left(Z_{1}\right)$. Für die Koeffizienten $a_{i} / c$, $i=1,2, \ldots$ in (3.16) gilt nach dem Bieberbachschen Flächensatz $\left|a_{i} / c\right| \leqq\left(R_{0}^{*}\right)^{i+1} i^{-1 / 2}$, $i=1,2, \ldots$ Es gibt ein $R_{2}>R_{0}$, so daß gilt

$$
2 R_{0}^{*} \leqq\left|H_{f}(z)\right| \text { für alle } z \text { mit }|z| \geqq R_{2} \text { und alle obigen } f
$$

(z. B. $\left.R_{2}=\max \left\{1,4 R_{0}^{*},\left[2\left(d_{0}+d_{1}\right)\right]^{p / 2}\right\}\right)$. Folglich gilt für alle oben genannten $f$ die Abschätzung

$$
\left|\frac{f(z)-a_{0}}{z}-c\right| \leqq|c||z|^{-1}\left|P_{0} h(z)+\frac{a_{1} / c}{z+P_{0} h(z)}+\ldots\right| \leqq|c| \cdot \delta(|z|)
$$

mit

$$
\delta(|z|)=\left(d_{0}+d_{1}\right)|z|^{-2 / p}+R_{0}^{*}|z|^{-1} \quad \text { für } \quad|z| \geqq R_{2} .
$$

Zusammen mit (3.17) folgt daraus

$$
\left|\frac{f(z)-f\left(z_{1}\right)}{z}-c\right| \leqq|c| \cdot\left[\left(d_{0}+d_{1}\right)|z|^{-2 / p}+\left(R_{0}^{*}+L\right) \cdot|z|^{-1}\right]
$$

für $|z| \geqq R_{2}$ und alle $z_{1} \in Z_{1}$. Für $|z|=R_{2}$ gilt folglich

$$
\left|f(z)-f\left(z_{1}\right)\right| \leqq|c| \cdot\left[\left(d_{0}+d_{1}\right) R_{2}^{1-(2 / p)}+R_{0}^{*}+L+R_{2}\right] .
$$

Da $f(z)-f\left(z_{1}\right)$ bei festem $z_{1}$ eine schlichte gebietstreue Abbildung ist mit $\infty \rightarrow \infty$, gilt (3.22) auch für alle $z \in G$ mit $|z| \leqq R_{2}$. Damit ist Satz 4 bewiesen.

Beweis von Satz 3. Durch die affine Hilfstransformation

$$
F(z)=\frac{1}{1-|\mu(\infty)|^{2}}(f(z)-b)-\frac{\mu(\infty)}{1-|\mu(\infty)|^{2}}(\overline{f(z)-b})+b
$$

entsteht aus jedem $f \in \mathscr{Q}(\mu, b)$ ein $F \in \mathscr{Q}\left(\mu_{0}, b\right)$ mit dem in (1.5) definierten $\mu_{0}$. Auf jedes $F \in \mathscr{Q}\left(\mu_{0}, b\right)$ und jedes $f \in \Sigma\left(\mu^{0}, b\right)$ ist Satz 4 mit $c_{F}=c_{f}=1$ und $z_{1}=b$ anwendbar, woraus sich Satz 3 ergibt.

Bemerkung 2. Eine zu Satz 3 völlig analoge Aussage gilt auch für $2(v, \mu, b)$ (siehe Bemerkung 1), wenn für die zugehörigen transformierten $v, \mu$ gefordert wird

$$
v, \mu \in L_{p_{0}} \quad \text { mit } \quad p_{0} \geqq 2 \text { und } \lim _{z \rightarrow \infty} v(z)=\lim _{z \rightarrow \infty} \mu(z)=0 .
$$

Bemerkung 3. Sei $\mu(\infty)=0$. Aus $\mu \in L_{p}, p>2$, folgt für $\mu_{1}(z)=\mu(1 / z)$ stets $\left(\mu_{1}(z) / z\right)|z|^{1-(4 / p)} \in L_{p}\left(E_{1}\right), E_{1}=\{|z|<1\}$, und damit nach der Hölderschen Ungleichung $\left(\mu_{1}(z) / z\right) \in L_{p^{*}}\left(E_{1}\right)$ für jedes $p^{*}<p$ mit $2 / p^{*}>1-2 / p$. Unter Verwendung eines Gedankens von B. Bojarski (siehe [5], S. 499 f) läßt sich folgender Satz beweisen. 
Satz 4'. Sei $\left(\mu_{1}(z) / z\right) \in L_{p *}\left(E_{1}\right), p^{*}>2$ und $C\left(p^{*}\right) \cdot$ ess $\sup _{|z|<1}\left|\mu_{1}\right|<1$. $Z u$ jeder quasikonformen Abbildung $g(z)$ von $E_{1}$ auf sich mit $g(0)=0$ und $\left|g_{\bar{z}}\right| g_{z} \mid \leqq$ $\left|\mu_{1}\right|$ für f.a. $z \in E_{1}$ existiert eine Konstante $c_{g} \neq 0, \neq \infty$, so daß

$$
\left|\frac{g(z)}{z}-c_{g}\right| \leqq c^{*}|z|^{1-\left(2 / p^{*}\right)} \text { für alle z mit } 0<|z|<1
$$

erfüllt ist mit einer positiven endlichen Konstanten $c^{*}>1$, die bei festem $\mu_{1}$ und festem $p^{*}$ unabhängig vom jeweiligen $g$ ist. Für $c_{g}$ gilt dabei

$$
1 / c^{*} \leqq\left|c_{g}\right| \leqq c^{*}
$$

(vgl. [7]). Wenn darüberhinaus $\mu_{1}(z) \rightarrow 0$ strebt für $z \rightarrow 0$, so braucht $p^{*}$ nur die Bedingung $p^{*}>2$ zu erfüllen.

Der Vorteil der oben angedeuteten Betrachtungsweise besteht darin, daß auch ohne die Zusatzvoraussetzung $\mu_{1}(z) \rightarrow 0$ für $z \rightarrow 0$ eine Aussage (3.23) zustande kommt, auch wenn man dann dort das $p^{*}$ nicht kennt. Dagegen ist der Übergang $\mathrm{zu}$ Voraussetzungen und Betrachtungsweisen, die zu einer Lipschitzbedingung (siehe Satz 6 unten) führen, hier nicht so zwanglos möglich wie bei den Voraussetzungen und Betrachtungsweisen, die Satz 4 zu Grunde liegen.

Wenn $\lim _{z \rightarrow 0} \mu_{1}(z)=0$ ist, so sind die Sätze 4 und $4^{\prime}$ in folgendem Sinne gleichwertig: Sind die Voraussetzungen des Satzes 4 primär gegeben, so kann die Ungleichung des Satzes 4 ,,in beliebig guter Näherung“" bezüglich der Exponenten mittels Satz 4' erhalten werden. Sind dagegen die Voraussetzungen des Satzes 4' primär gegeben, so kann (3.23) mittels Satz 4 ,,in beliebig guter Näherung“ erhalten werden (denn dann ist $\mu(z)=\mu_{1}(1 / z) \in L_{p}(\{|z|>1\})$ für jedes $p$ mit $\left.2 / p<1-2 / p^{*}\right)$.

\section{Der Fall $\left(L^{q}\right)$}

Eine weitere ,Verbesserung“ der Verhältnisse in $\mathscr{2}(\mu, b), \Sigma(\mu, b)$ tritt ein bei Übergang von $\left(L_{p}\right) \mathrm{zu}\left(L^{q}\right)$, d. h. bei Absinken von $p_{0}$ unter 2. Dann gilt nämlich folgender

Satz 5. Die Funktion $\mu(z)$ erfülle die Voraussetzung $\left(L^{q}\right)$.

(I) Es existiert eine feste endliche Konstante $c_{3}$, so daß für alle $f(z)=z+$ $\mu(\infty) \bar{z}+\varepsilon_{f}(z) \in \mathscr{Q}(\mu, b)$ gilt $\left|\varepsilon_{f}(z)\right| \leqq c_{3}$ für alle $z \in G$.

(II) Für jedes $f \in \mathscr{Q}(\mu, b)$ existiert der Grenzwert $\lim _{z \rightarrow \infty} \varepsilon_{f}(z) \equiv a(f)$.

(III) Die Menge $\mathscr{Q}(\mu)=\{f(z)-a(f): f(z) \in \mathscr{Q}(\mu, b)\}$ ist kompakt in sich. Eine quasikonforme Abbildung $f$ von $G$ gehört genau dann zu $\mathscr{Q}(\mu)$, wenn sie (1.2), (1.3) erfüllt und eine Entwicklung $f(z)=z+\mu(\infty) \bar{z}+\varepsilon_{f}(z)$ mit $\lim _{z \rightarrow \infty} \varepsilon_{f}(z)=0$ besitzt. 
(IV) Wenn $\mu$ zusätzlich $\mu(\infty)=0$ erfüllt, so ist die Menge $\Sigma(\mu)$ der quasikonformen Abbildungen $f$, die (1.2) erfüllen und eine Entwicklung $f(z)=z+\varepsilon_{f}(z)$ mit $\lim _{z \rightarrow \infty} \varepsilon_{f}(z)=0$ besitzen, kompakt in sich.

Der Beweis von Satz 5 ergibt sich wieder aus einer Verschärfung des (erweiterten) Teichmüller-Wittichschen Verzerrungssatzes, nämlich dem

Satz 6. Die Funktion $\mu(z)$ erfülle (1.1), (1.8) und $\mu(\infty)=0$. Eine beschränkte Menge $Z_{1} \subset G$ sei beliebig, aber fest vorgegeben. Dann gibt es eine feste endliche positive Konstante $c_{4}$ und $z u$ jeder quasikonformen $f(z)$ von $G$ mit $f(\infty)=\infty$, die (1.2) erfüllt, eine endliche Konstante $c_{f} \neq 0$, so daß gilt

$$
\left|\frac{f(z)-f\left(z_{1}\right)}{z}-c_{f}\right| \leqq\left|c_{f}\right| \cdot c_{4} \cdot|z|^{-1} \text { für alle } z \in G \text { und alle } z_{1} \in Z_{1} \text {. }
$$

Beweis von Satz 6. Wegen der Stetigkeit von $C(s)$ und $C(2)=1$ gibt es ein festes $\delta>0$ mit

(4.1) $C(s) \cdot q_{0} \leqq k<1$ für alle $s$ aus dem abgeschlossenen Intervall $[2-\delta, 2+\delta]$, $k$ eine zuvor beliebig, aber fest gewählte Konstante mit $q_{0}<k<1$. Seien nun $p, q$ zwei feste Zahlen mit $2<p \leqq 2+\delta, 2-\delta \leqq q<2$, und ohne Beschränkung der Allgemeinheit (siehe (1.10)) stimme dieses $q$ mit dem in (1.8) genannten überein.

Sei $L^{p, q}=L_{p} \cap L_{q}$. Für $h \in L^{p, q}$ ist nicht nur $P_{0}$, sondern auch

$$
\operatorname{Ph}(z)=-\frac{1}{\pi} \int \frac{h(\zeta)}{\zeta-z} d \sigma_{\zeta}
$$

definiert, und es gilt $P_{0} h(z)=P h(z)-P h(0) . \quad L^{p, q}$ ist ein Banachraum mit der Norm $\|h\|_{L^{p, q}}=\max \left\{\|h\|_{L_{p}},\|h\|_{L_{q}}\right\}$. Zerlegt man das Intagrationsgebiet rechts in (4.2) in $\{|\zeta-z|<R\}$ und $\{|\zeta-z|>R\}$ mit irgendeinem reellen $R>0$, so liefert die Höldersche Ungleichung die Abschätzung

$$
|P h(z)| \leqq K_{p, q}\|h\|_{L^{p, q}} \text { für alle } z
$$

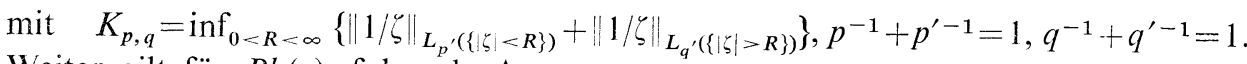
Weiter gilt für $P h(z)$ folgende Aussage.

(4.4) Für jedes $h \in L^{p, q}$ mitt $\infty>p>2,1<q<2$ ist $\lim _{z \rightarrow \infty} P h(z)=0$.

Beweis. Sei $|z|=2 t>2, R(t)=\{\zeta: t<|\zeta|<3 t\}, K(z, s)=\{\zeta:|\zeta-z|<s\}$. Mit $q^{\prime}=$ $q /(q-1)>2,1<p^{\prime}=p /(p-1)<2$ gilt dann

$$
\begin{aligned}
& \left|\int \frac{h(\zeta)}{\zeta-z} d \sigma_{\zeta}\right| \leqq\left|\int_{\{|\zeta|<t\}}\right|+\left|\int_{R(t) \backslash K(z, t)}\right|+\left|\int_{K(z, t) \backslash K(z, 1)}\right|+\left|\int_{K(z, 1)}\right|+\left|\int_{\{|\zeta|>3 t\}}\right| \\
& \leqq\|h\|_{L_{q}} \cdot\left(\pi t^{2}\right)^{1 / q^{\prime}} \cdot t^{-1}+\|h\|_{L_{q}} \cdot\left(8 \pi t^{2}\right)^{1 / q^{\prime}} \cdot t^{-1}+\|h\|_{L_{q}(\{|\zeta|>t\})} \cdot\|1 / \zeta\|_{L_{q^{\prime}}(\{|\zeta|>1\})} \\
& +\|h\|_{L_{p}(\{|\zeta|>t\})} \cdot\|1 / \zeta\|_{I_{p^{\prime}}(\{|\zeta|<1\})}+\|h\|_{L_{q}(\{|\zeta|>3 t\})} \cdot\|1 / \zeta\|_{L_{q^{\prime}}(\{|\zeta|>1\})} .
\end{aligned}
$$

Für $t \rightarrow \infty$ folgt dann die Behauptung (4.4). (Für die in [12], S. 37 betrachtete Klasse 
$L_{p, 2}(E)$ mit $p>2$ gilt eine schärfere Aussage als (4.4). Natürlich ist $L_{p, 2}(E) \subset$ $L^{p, q}$ für $q>p /(p-1)$, jedoch $L^{p, q} \leftarrow L_{p, 2}(E)$ für alle $p>2, q \geqq 1$, wie einfache Beispiele zeigen.)

Sei nun wieder $f$ irgendeine quasikonforme Abbildung von $G$ mit $f(\infty)=\infty$, die (1.2) erfüllt. Die Funktion $\mu_{f}(z)=f_{\bar{z}} / f_{z}$ für f. a. $z \in G$ und $=0$ sonst gehört dann auch zu $L^{p, q}$, und

$$
H_{f}(z)=z+P h(z)
$$

ist wieder eine schlichte Lösung von (3.9) in der ganzen $z$-Ebene mit $H_{f}(\infty)=\infty$, wobei $h$ Lösung von

$$
h=\mu_{f}+\mu_{f} T h \quad \text { mit } \quad\|h\|_{L^{p, q}} \leqq \frac{1}{1-k}\left\|\mu_{f}\right\|_{L^{p, q}} \leqq \frac{1}{1-k}\|\mu\|_{L^{p, q}}
$$

in $L^{p, q}$ ist. Folglich gilt für jedes obengenannte $f(z)$

$$
f(z)=\omega_{f} \circ H_{f}(z)
$$

mit einem $\omega_{f}$ wie in (3.14). Die $H_{f}$ bilden wegen (4.5), (4.3), (4.6) wieder eine kompakte Menge in der Menge der schlichten $Q$-quasikonformen Abbildungen der vollen Ebene auf sich. Zum Definitionsbereich der $\omega_{f}$ in (4.7) gehört stets $\left\{|H|>R_{0}^{*}\right\}$ mit $R_{0}^{*}=R_{0}+K_{p, q} \cdot(1 /(1-k))\|\mu\|_{L^{p, q}}$. Der Rest des Beweises von Satz 6 verläuft völlig analog zum Beweis von Satz 4 .

Beweis von Satz 5. Im Hinblick auf (1.4') genügt es natürlich wieder, Satz 5 im Falle $\mu(\infty)=0$ zu beweisen. Die Behauptung (III) folgt unmittelbar aus (I), (II) und Satz 1, (I) folgt sofort aus Satz 6 mit $c_{f}=1, z_{1}=b$. Die Behauptung (II) ergibt sich aus (4.7) zusammen mit (3.14) und (4.4). Die Behauptung (IV) folgt daraus, da $\beta$ die $f \in \Sigma(\mu, b)$ bei $\mu(\infty)=0$ dieselben Abschätzungen und eine Darstellung (4.7) gestatten wie die $f \in \mathscr{Q}(\mu, b)$ bei $\mu(\infty)=0$. Folglich gibt es zu jedem $f \in \Sigma(\mu, b)$ auch eine Konstante $a(f)=a_{0 f}$ (siehe $a_{0}$ in (3.14)), so da $\beta f-a_{0 f} \in \Sigma(\mu)$ ist und umgekehrt, und die $a_{0 f}$ sind wieder gleichmäßig beschränkt. Damit ist Satz 5 bewiesen.

Die Klassen $2(\mu)$ und $\Sigma(\mu)$ (bei $\Sigma(\mu)$ natürlich $\mu(\infty)=0$ ) besitzen große Ähnlichkeit mit der Klasse $\Sigma(G)$ der hydrodynamisch normierten konformen Abbildungen von $G$. Das Abklingen von $\mu-\mu(\infty)$ unter der Bedingung $\left(L^{q}\right)$ für $z \rightarrow \infty$ führt zu solch „stabilen Verhältnissen“ bei den $f \in \mathscr{Q}(\mu, b)$ und den $f \in \Sigma(\mu, b)$, da $\beta$ man die Forderung $f(b)=b$ (oder eine ähnliche Forderung) hier wie im „Idealfall“ $\mu \equiv 0$ durch zusätzliche Forderungen an die asymptotischen Entwicklungen ersetzen kann, was zu den Klassen $2(\mu)$ und $\Sigma(\mu)$ führt. Diese Ähnlichkeit von $2(\mu)$ und $\Sigma(\mu)$ mit $\Sigma(G)$ legt es nahe, die $f$ aus $2(\mu)$ und die $f$ aus $\Sigma(\mu)$ verallgemeinert hydrodynamisch normiert zu nennen.

Bemerkung 4. Eine zu Satz 5 völlig analoge Aussage gilt natürlich auch für $\mathscr{Q}(v, \mu, b)$ (siehe Bemerkung 1), wenn die zugehörigen transformierten $v, \mu$ beide zu $L_{q}$ mit $1 \leqq q<2$ gehören. 


\section{Literatur}

[1] AhLfors, L. V.: Lectures on quasiconformal mappings. - D. Van Nostrand Company, Inc., Princeton, New Jersey-Toronto-New York-London, 1966.

[2] AhLfors, L. V., and L. Bers: Riemann's mapping theorem for variable metrics. - Ann. of Math. 72, 1960, 385-404.

[3] BeLInskĭ̌, P. P.: Das Verhalten einer quasikonformen Abbildung in einem isolierten Punkt. Doklady Akad. Nauk SSSR (N. S.) 91, 1953, 709-710. (Russisch).

[4] Bieberbach, L.: Einführung in die konforme Abbildung. - Sammlung Göschen Bd. 768, 768a. Berlin, 1967.

[5] Bojarski, B. V.: Generalized solutions of a system of differential equations of first order and elliptic type with discontinuous coefficients. - Mat. Sb. N. S. 43 (85), 1957, 451-503. (Russian).

[6] KüHnaU, R. und H. Renelt: Ein Existenzbeweis für schlichte Lösungen linearer elliptischer Differentialgleichungssysteme durch eine Integralgleichung. - Math. Nachr. 79, 1977, 225-232.

[7] Lehto, O.: On the differentiability of quasiconformal mappings with prescribed complex dilatation. - Ann. Acad. Sci. Fenn. Ser. A I 275, 1960, 1-28.

[8] Lehto, O., and K. I. VirTANen: Quasiconformal mappings in the plane. - 2. Auflage, BerlinHeidelberg-New York, 1973.

[9] Schiffer, M.: Analytical theory of subsonic and supersonic flows. - Handbuch der Physik Bd. IX, herausgegeben von S. Flügge, Berlin-Göttingen-Heidelberg, 1960, 1-161.

[10] Strebel, K.: Ein Konvergenzsatz für Folgen quasikonformer Abbildungen. - Comment. Math. Helv. 44, 1969, 469-475.

[11] TeICHMÜLLER, O.: Untersuchungen über konforme und quasikonforme Abbildungen. - Deutsche Math. 3, 1938, 621-678.

[12] Vekua, I. N.: Verallgemeinerte analytische Funktionen. - Akademie-Verlag, Berlin, 1963.

[13] Wiтtich, H.: Zum Beweis eines Satzes über quasikonforme Abbildungen. - Math. Z. 51, 1949, $278-288$

Martin-Luther-Universität Halle-Wittenberg

Sektion Mathematik

Universitätsplatz 6

DDR-4020 Halle an der Saale

Deutsche Demokratische Republik

Eingegangen am 14. Juni 1979 